\title{
331.
}

\section{ANALYTICAL THEOREM RELATING TO THE FOUR CONICS INSCRIBED IN THE SAME CONIC AND PASSING THROUGH THE SAME THREE POINTS.}

[From the Philosophical Magazine, vol. xxvir. (1864), pp. 42, 43.]

ImAGINE the four conics determined, and, selecting at pleasure any three of them, let their chords of contact with the given conic be taken for the axes of coordinates, or lines $x=0, y=0, z=0$; then, taking for the equation of the given conic

$$
U=\left(a, b, c, f, g, h \gamma(x, y, z)^{2}=0,\right.
$$

the equations of the selected three conics must be of the form $U+l x^{2}=0, U+m y^{2}=0$, $U+n z^{2}=0$, where $l, m, n$ are to be determined in such manner that these conics may have three common points; the resulting values of $l, m, n$, and of the coordinates of the three common points, that is, the three given points, will of course be functions of the coefficients $(a, b, c, f, g, h)$; and the equation of the fourth conic will be of the form $U+(i x+j y+k z)^{2}=0$.

There is no difficulty in carrying out the investigation: it is found that the coordinates of the given points must be taken to be

$$
(-f, g, h) ;(f,-g, h) ;(f, g,-h)
$$

respectively, and that, writing as usual

$$
K=a b c-a f^{2}-b g^{2}-c h^{2}+2 f g h,
$$


the equations of the four conics are

$$
\begin{aligned}
& U+(K-a b c) \frac{x^{2}}{f^{2}}=0, \\
& U+(K-a b c) \frac{y^{2}}{g^{2}}=0, \\
& U+(K-a b c) \frac{z^{2}}{h^{2}}=0 \\
& U+(K-a b c)\left(\frac{x}{f}+\frac{y}{g}+\frac{z}{h}\right)^{2}=0 .
\end{aligned}
$$

It is in fact easy to verify directly that each of these conics passes through the three given points; but the equations may also be exhibited in the form proper for putting this in evidence. Putting for shortness

$$
X=\frac{y}{g}+\frac{z}{h}, \quad Y=\frac{z}{h}+\frac{x}{f}, \quad Z=\frac{x}{f}+\frac{y}{g},
$$

the equations of the sides of the triangle formed by the given points are $X=0, Y=0, Z=0$, and the foregoing equations of the four conics may be expressed in the form

$$
\begin{array}{rr}
\left(-b g^{2}-c h^{2}+2 f g h\right) Y Z+ & b g^{2} \cdot Z X+ \\
a f^{2} \cdot Y Z+\left(-c h^{2}-a f^{2}+2 f g h\right) Z X+ & c h^{2} \cdot X Y=0 \\
a f^{2} \cdot Y Z+ & b g^{2} \cdot Z X+\left(-a f^{2}-b g^{2}+2 f g h\right) X Y=0 \\
\left(-b g^{2}-c h^{2}+2 f g h\right) Y Z+\left(-c h^{2}-a f^{2}+2 f g h\right) Z X+\left(-a f^{2}-h g^{2}+2 f g h\right) X Y=0
\end{array}
$$

which is $t^{*}$ required form.

Cambridge, November 28, 1863. 